\title{
Lumbar Interbody Fusion: Techniques, Pearls and Pitfalls
}

\author{
Young-Hoon $\mathrm{Kim}^{1}$, Kee-Yong $\mathrm{Ha}^{2}$, Kee-Won Rhyu ${ }^{3}$, Hyung-Youl Park ${ }^{4}$, \\ Chang-Hee Cho ${ }^{1}$, Hun-Chul Kim ${ }^{1}$, Hyo-Jin Lee ${ }^{1}$, Sang-Il Kim ${ }^{1}$ \\ ${ }^{1}$ Department of Orthopedic Surgery, Seoul St. Mary's Hospital, College of Medicine, The Catholic University of Korea, Seoul, Korea \\ ${ }^{2}$ Department of Orthopedic Surgery, Kyung Hee University Hospital at Gangdong, Seoul, Korea \\ ${ }^{3}$ Department of Orthopedic Surgery, St. Vincent's Hospital, College of Medicine, The Catholic University of Korea, Suwon, Korea \\ ${ }^{4}$ Department of Orthopedic Surgery, Eunpyeong St. Mary's Hospital, College of Medicine, The Catholic University of Korea, Seoul, Korea
}

Lumbar interbody fusion (LIF) is an effective and popular surgical procedure for the management of various spinal pathologies, especially degenerative diseases. Currently, LIF can be performed with posterior, transforaminal, anterior, and lateral approaches by open surgery or minimally invasive surgery (MIS). Each technique has its own advantages and disadvantages. In general, posterior LIF is a well-established procedure with good fusion rates and low complication rates but is limited by the possibility of iatrogenic injury to the neural structures and paraspinal muscles. Transforaminal LIF is frequently performed using an MIS technique and has an advantage of reducing these iatrogenic injuries. Anterior LIF (ALIF) can restore the disk height and sagittal alignment but has inherent approach-related challenges such as visceral and vascular complications. Lateral LIF and oblique LIF are performed using an MIS technique and have shown postoperative outcomes similar to ALIF; however, these approaches carry a risk of injury to psoas, lumbar plexus, and vascular structures. Herein, we provide a detailed description of the surgical procedures of each LIF technique. We shall then consider the pearls and pitfalls, as well as propose surgical indications and contraindications based on the available evidence in the literatures.

Keywords: Lumbar vertebrae; Intervertebral disc; Spinal fusion; Minimally invasive surgery; Interbody fusion

\section{Introduction}

Lumbar interbody fusion (LIF) has been proven as an excellent treatment option for various spinal pathological conditions, such as degenerative disk disease, deformity, infection, tumor, and trauma [1]. LIF comprises discectomy, endplate preparation, and placement of different kinds of implants. The main objective of LIF is to restore the intervertebral space and stabilize the segments with proper height and lordosis. In the early 20th century, Russell Hibbs and Fred Albee introduced spinal fusion procedures with a bridge of spinous process and wiring techniques. Thereafter, researchers reported instrumented posterolateral fusion (PLF) with facet screws [1]. However, pseudarthrosis after PLF was developed with unacceptable incidences. LIF had been introduced to reduce the rates of pseudarthrosis and other postoperative complications. Since the first description of LIF using a posterior

\footnotetext{
Received Sep 17, 2020; Accepted Sep 18, 2020

Corresponding author: Sang-Il Kim

Department of Orthopedic Surgery, Seoul St. Mary's Hospital, College of Medicine, The Catholic University of Korea, 222 Banpo-daero, Seocho-gu, Seoul 06591, Korea

Tel: +82-2-2258-6775, Fax: +82-2-535-9837, E-mail: sang1kim81@gmail.com
} 


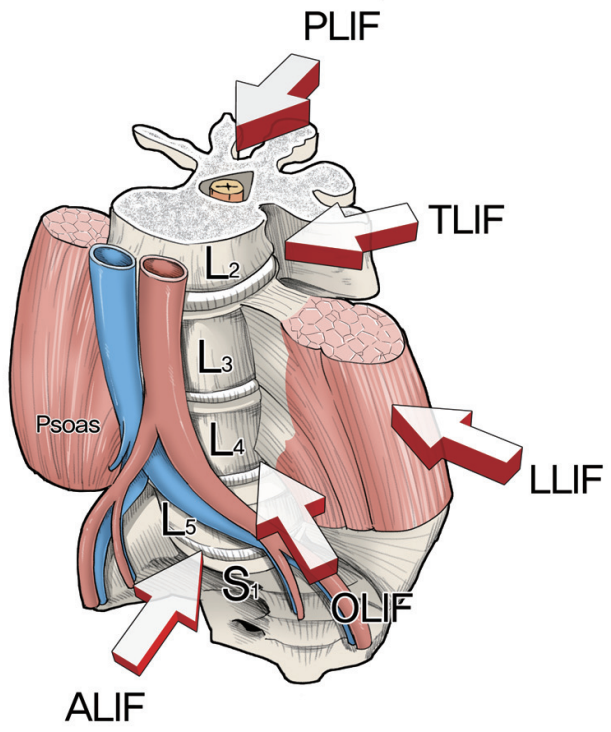

Fig. 1. The five lumbar interbody fusion techniques are shown: PLIF, TLIF, LLIF, OLIF, and ALIF. PLIF, posterior lumbar interbody fusion; TLIF, transforaminal lumbar interbody fusion; LLIF, lateral lumbar interbody fusion; OLIF, oblique lumbar interbody fusion; ALIF, anterior lumbar interbody fusion.

approach by Briggs and Milligan [2] in 1944, researchers have developed many other techniques, such as posterior lumbar interbody fusion (PLIF), transforaminal lumbar interbody fusion (TLIF), anterior lumbar interbody fusion (ALIF), lateral lumbar interbody fusion (LLIF), and oblique lumbar interbody fusion (OLIF) (Fig. 1). So far, there is no clear and high-level evidence that one of these approaches holds clinical superiority over the other [3]. Each has its own advantages and disadvantages.

Historically, Burns and Capener reported the first interbody fusion in the 1930s [1]. They treated spondylolisthesis using anterior approaches. In 1944, Briggs and Milligan [2] became the first researchers to describe PLIF, which was evolved by Cloward [4] in the early 1950s. In 1988, Steffee and Sitkowski [5] proposed the "load sharing" concept using the anterior interbody support combined with pedicle screw fixation. Harms and Rolinger [6] introduced TLIF as an alternative to PLIF in 1982 [7]. Following technical advances in surgical instruments, minimally invasive surgery (MIS) has received considerable interest as an alternative to open surgery. These traditional operations can be performed using MIS approaches [8]. To avoid the disruption of the posterior spinal structures, MIS-ALIF, LLIF, and OLIF are recently gaining popularity.

The purpose of this review is to present the surgical techniques of each LIF, as well as provide the pearls and pitfalls along with their specific indications/contraindications.

\section{Posterior Lumbar Interbody Fusion}

\section{Surgical techniques}

PLIF was one of the first techniques employed for LIF. Surgical access to the intervertebral disc is achieved via a posterior approach. The procedure begins with proper positioning, which is essential in obtaining the favorable outcomes. The patient rests in the prone position on an operation table in hypertension to help create lumbar lordosis. Several positions such as knee-chest position or a Jackson table are also available. An important aspect of either position is the elimination of the abdominal pressure; this action decompresses the epidural venous plexus and helps in reducing the intraoperative bleeding. The classic PLIF procedure includes the application of an open midline approach to subperiosteally expose the spinous process, laminae, facets, and transverse process of the involved segments. The pedicle screws are placed either before or after a laminectomy. If placed first, pedicle screws can be used to distract the intervertebral space during PLIF. A wide laminectomy is usually performed to resect the caudal two-thirds of the rostral vertebra's lamina $[4,9]$. The superior edge of the rostral vertebra's lamina should be preserved to maintain the posterior ligamentous complex to the adjacent rostral vertebra. Bony resection can be extended laterally to perform the complete or partial excision of the inferior and superior articular processes. The facets should always be excised completely to correct deformity [10]. Once ligamentum flavum is removed and the thecal sac is exposed, the thecal sac and traversing nerve root are retracted at both sides medially to access the intervertebral disc space. Thereafter, annulotomy, discectomy, and endplate preparation are serially performed. Only the cartilaginous endplates, but not the bony endplates, should be removed to prevent the graft subsidence. In most cases, the anterior annulus prevents the anterior protrusion of implants, but the anterior annulus is sometimes deficient, especially in spondylolisthesis. It is helpful to mark the shaver or the curettes so that they are not inserted past a depth of $30 \mathrm{~mm}$ [10]. After cleaning the intervertebral space, it is measured using trial spacers and the cage or bone graft is inserted into the interspace through each of the two annulotomy procedures. Finally, 
supplemental pedicle screw and rod constructs are applied.

\section{Pearls and pitfalls}

PLIF is a traditional lumbar approach that allows access to both the anterior and posterior columns with one incision. PLIF is performed using the most common posterior approach, which is familiar to most of the spine surgeons. Another advantage of PLIF is the excellent visualization of neural structures, which allows bilateral decompression $[11,12]$. However, PLIF also has inherent disadvantages. Spine surgeons must remember the existence of anatomical variations, such as a spina bifida occulta or a usually wide interlaminar space. These abnormalities should be identified before the surgery to avoid inadvertent injury to the dura or nerve roots. PLIF inevitably causes paraspinal muscle injury because of the surgical approach and prolonged muscle retraction [13]. Additionally, PLIF requires a more retraction of thecal sac and nerve roots to achieve an adequate surgical exposure of intervertebral space [1417]. Because of this concern, PLIF is usually limited to the lower lumbar level (L3-S1) to avoid damage to the cord. Nevertheless, this retraction injury of nerve root may induce fibrosis and chronic radiculopathy. Additionally, the retraction of dura may pose an increased risk of durotomy, especially during the revision surgery [18].

Current treatment guidelines are mainly based on level III studies, and there is a paucity of level I or II studies. Acceptable indications for PLIF include degenerative disk disease, lumbar segment instability, spondylolisthesis, degenerative scoliosis, pseudarthrosis, and recurrent disk herniation $[9,19]$. The neural structures including dura and nerve roots should be mobilized to access the disk space. Therefore, severe epidural fibrosis is a contraindication to PLIF. Other contraindications may include arachnoiditis, active infections, and severe osteoporosis.

\section{Transforaminal Lumbar Interbody Fusion}

\section{Surgical techniques}

In 1982, Harms and Rolinger [6] introduced TLIF as an alternative to PLIF for the management of degenerative spine diseases [7]. In the early 2000s, Foley et al. [20,21] introduced MIS-TLIF as a possible alternative to reduce the approach-related complications. The basic concept of

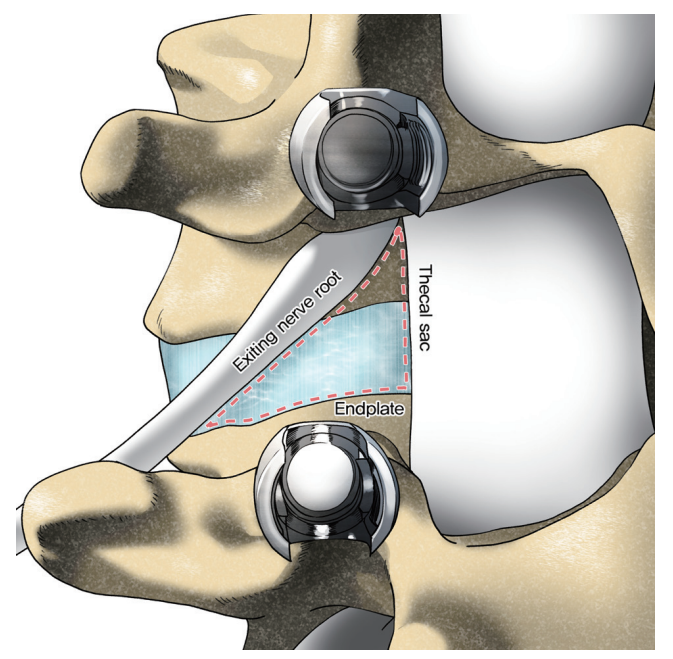

Fig. 2. Kambin's triangle is a safe transforaminal corridor to the intervertebral disc, bordered medially by the traversing nerve root and thecal sac, anteriorly by the exiting nerve root, and inferiorly by the cranial endplate of the caudal vertebra.

TLIF is accessing the intervertebral disc space on a more lateral trajectory than PLIF [22]. The patient positioning is same as those for PLIF. In a traditional open TLIF, a posterior midline incision is introduced for the subperiosteal exposure of the spinous process, laminae, facets, and transverse process of the involved segments. MIS-TLIF is performed based on a Wiltse-type approach using an expandable tubular retractor to access the posterior elements. A $3-\mathrm{cm}$ longitudinal incision is made $2-3 \mathrm{~cm}$ lateral to the midline. To access the intervertebral space, the pars interarticularis is identified and resected. Thereafter, facetectomy of the superior and inferior facets of the levels to be fused is performed, thereby providing access to the Kambin's triangle (Fig. 2). The traversing nerve root is retracted medially, and discectomy is then performed in a standard fashion, followed by endplate preparation. Next, a trial may be used to verify the cage size, and intervertebral cage with morselized autologous or allogeneic bone graft is placed within the disk space. Depending on the preference of the surgeon, the pedicle screws are placed either before or after the TLIF procedure.

\section{Pearls and pitfalls}

TLIF can provide a fusion of $360^{\circ}$ with a single incision. However, the most significant advantage of TLIF, compared to PLIF, is achieving a more lateral access to the disk, thus reducing retraction on the neural elements. TLIF can be safely conducted even at the upper lumbar 
segments. Therefore, it appears that in TLIF, the lesser dural exposure may have a lower risk of durotomy than PLIF [23]. Compared to PLIF, TLIF can cause less damage on the posterior ligamentous complex and preserve biomechanical stability. In the biomechanical studies, TLIF seems to show comparable results with PLIF $[24,25]$. Additionally, TLIF may be preferable in the revision cases, because the lateral access may avoid the postoperative scar tissue on the posterior approach [26-28]. Recently, MIS techniques have gained popularity because these techniques may show favorable results such as reduced blood loss and muscle injury, operative time, and an early postoperative recovery than the traditional open techniques [23,29-34].

The pitfall is that TLIF, especially with open techniques, may cause considerable paraspinal muscle injury associated with prolonged muscle retraction. Another theoretical pitfall of TLIF is that it is impossible to completely remove the disk materials because only a unilateral approach is performed [10,35]. McAfee et al. [36] showed that the fusion rate following ALIF decreased in the patients with incomplete discectomy than in the patients with complete discectomy. Despite this concern, no prospective randomized comparison has been reported. Although MIS-TLIF may reduce postoperative morbidities, it seems to have the learning curve [37-39].

Indications and contraindications for TLIF are similar to those for PLIF. Indications include all the degenerative disk diseases, lumbar segment instability, spondylolisthesis, degenerative scoliosis, pseudarthrosis, and recurrent disk herniation. Contraindications include epidural scar, arachnoiditis, active infections, and severe osteoporosis. Additionally, nerve root anomaly is a relative contraindication because it can increase the risk of iatrogenic nerve injury in the nerve roots.

\section{Anterior Lumbar Interbody Fusion}

\section{Surgical techniques}

A detailed patient evaluation is required before the consideration of ALIF procedure. Additionally, a history for abdominal surgery or radiation therapy should also be obtained. It is recommended that the area of interest should be evaluated by magnetic resonance imaging and/ or computed tomography scans preoperatively to carefully review the vascular structures along with the lumbosacral

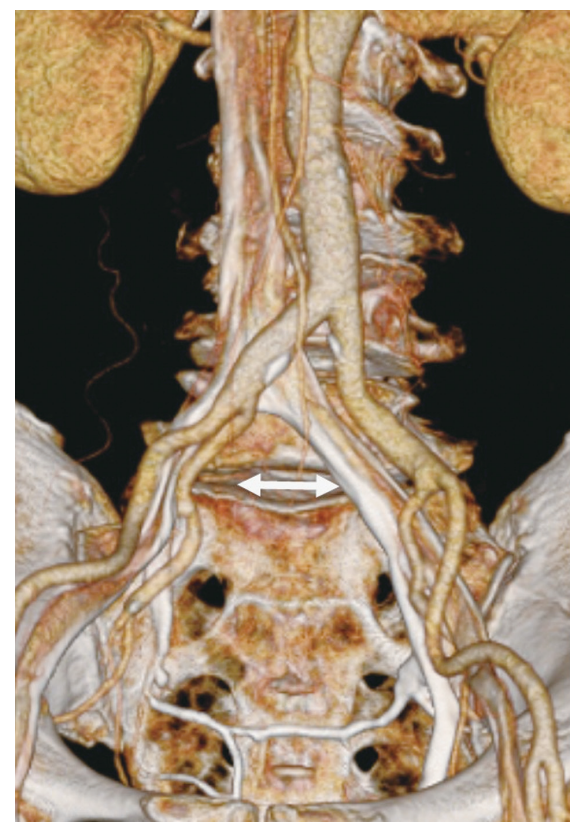

Fig. 3. Three-dimensional computed tomography angiography can help evaluate the accessibility for anterior lumbar interbody fusion.

spine (Fig. 3) [40-42]. Both retroperitoneal and transperitoneal approaches are available for ALIF [43]. Because the transperitoneal approach has been associated with higher rates of bowel injury, ileus, and retrograde ejaculation, and is limited at the L5-S1 segment, many spine surgeons favor a retroperitoneal approach [43-45]. Therefore, retroperitoneal approach will be discussed in this review.

The patient is kept in a true supine position for L4-5 and L5-S1 levels. It may be helpful to position the patient slightly in a right lateral decubitus position to access the multiple levels of the lumbar spine, especially higher levels. The usual approach is initiated from the patient's left side, since the aorta is more robust than the vena cava. The hip-flexed position is helpful in relaxing the iliopsoas during retraction. Incisions may vary according to the levels. The skin incision may be either medial horizontal (Pfannenstiel) for L5-S1 or medial vertical. Longitudinal incision may present a higher risk of rectus abdominis denervation and atonia. With careful inspection and gentle finger dissection, the transversalis fascia and arcuate line are identified and detached from the lateral abdominal wall. Once the transversalis fascia is incised, the peritoneal sac is moved medially to access the retroperitoneal space via blunt dissection. If the peritoneum is opened, then it should be immediately closed using absorbable 3-0 or 4-0 sutures to prevent postoperative hernia. The landmark to 
be palpated is the psoas muscle, along with the pulsation of the common iliac artery. The next landmark is L5-S1 disk and sacral promontory. At L5-S1 level, the middle sacral vessels should be cauterized. In front of L5-S1 disk, the autonomic nerves exist and may be damaged by monopolar electrocautery, thereby leading to autonomic dysfunction. At L4-5 level, the left common iliac artery and vein must be mobilized from left to right. Additionally, the left iliolumbar vein can be encountered at L5. It should be ligated before mobilizing the left common iliac vein to avoid tethering or massive bleeding $[45,46]$.

After the confirmation of the correct level, anterior annulotomy, discectomy, and endplate preparation are performed serially. The excessive depth of discectomy should be avoided. If the dorsal annulus fibrosus is violated, then epidural bleeding or a cerebrospinal fluid leak can occur. Finally, a trial may be used to verify the cage size, and intervertebral cage with morselized autologous or allogeneic bone graft is placed within the disk space.

\section{Pearls and pitfalls}

The benefits of ALIF include a less traumatic access than posterior approaches, resulting in less postoperative pain and shorter hospital stays [47-49]. The posterior elements of the spine such as ligaments and muscles may be preserved [50]. However, the opposite results, complication rates including surgical site infection, and nerve injury seem to be lower after ALIF [48,51,52]. Above all, ALIF can use large interbody grafts, which provide a significant biomechanical advantage than other LIFs [53]. Recently, the importance of sagittal balance has been increasingly recognized among researchers. The potential to gain a sagittal alignment may increase with more lordotic cages placed at the lower lumbar spine [54-58]. This action can be achieved by ALIF in the lower lumbar spine [57,59].

The pitfalls of ALIF include the requirement of great vessel mobilization, which may lead to deep vein thrombosis and a direct injury [60]. There are possible risks for abdominal organ and ureter injury, along with incisional hernia [60]. Injury to hypogastric plexus can occur and result in retrograde ejaculation in up to $45 \%$ of men $[52,61,62]$. Approach-related sympathectomy symptoms, such as vasodilation in the ipsilateral leg, usually occur, but almost always resolve spontaneously in the first three to six months after surgery $[48,63]$.

Proper patient selection is an important prerequisite for a successful ALIF procedure. Surgical indications for ALIF procedure are degenerative disk disease, failed posterior fusion, or postoperative spondylodiscitis. Additionally, ALIF may be suitable for the surgical management of sagittal plane deformities. Contraindications of ALIF are morbid obesity, prior abdominal surgery, prior radiation therapy, and severe aortic atherosclerosis.

\section{Lateral Lumbar Interbody Fusion}

\section{Surgical techniques}

In 2006, Ozgur et al. [64] were the first ones to describe the MIS-LLIF technique, also called transpsoas. The corridor for LLIF is limited superiorly by the 11th or 12th rib and inferiorly by the iliac crest. With LLIF, the access is possible at from L1-2 to L4-5 [10]. Before positioning, the appropriate electromyography (EMG) monitoring leads should be placed. The table must be radiolucent and have capabilities to break Trendelenburg, reverse Trendelenburg, and tilt positions. The LLIF procedure begins with the patient lying in either the left or right lateral decubitus position. The hip and knees are flexed. The table may be bent to position the iliac crest away for accessing L4-5. The patients are taped carefully. After the completion of initial positioning, intraoperative fluoroscopy is used to establish a true anteroposterior and lateral plane to the disk. A lateral incision is introduced and the abdominal musculature is bluntly dissected. The retroperitoneal space is identified by the presence of a yellow fat tissue deep into the transversalis muscle. Blunt finger dissection is used to sweep the peritoneal sac anteriorly (Fig. 4). Posteriorly, the quadratus lumborum is palpated; the medial movement allows the surgeon to encounter the transverse process. Medial to the transverse process, the round psoas muscle is palpated. EMG monitoring is used within psoas to avoid lumbar plexus [65]. Lateral fluoroscopy is utilized to ensure that the correct level is being accessed and the trajectory will be in a safe zone (Fig. 5) [66]. Sequential dilators are placed on the psoas muscle, and the tubular retractor is introduced. After the dissection of muscle fibers, the disk space is identified and annulotomy is performed. A Cobb elevator is placed into the disk space and malleted under fluoroscopy until the contralateral annulus is disrupted. A box cutter disc shaver is used to perform discectomy. During the steps of using a Cobb elevator and a shaver, it is essential to avoid end- 


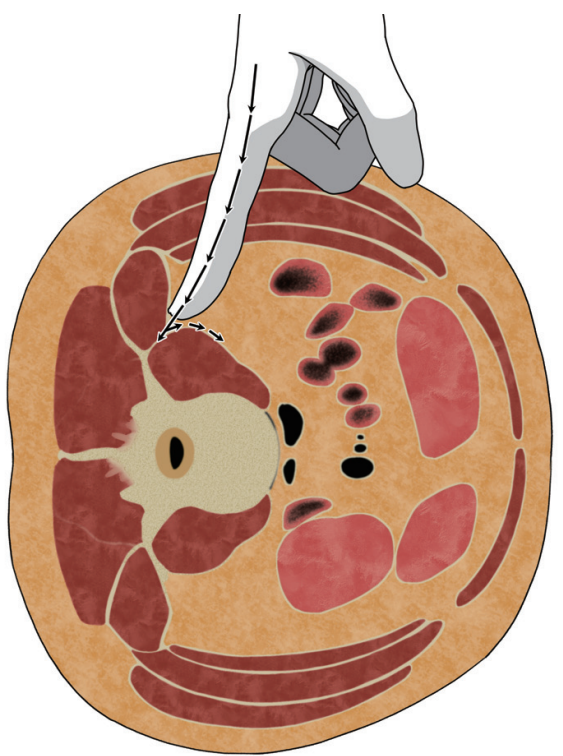

Fig. 4. A schematic illustration of blunt finger dissection during lateral lumbar interbody fusion. All of the peritoneal contents are swept anteriorly to avoid visceral damages.
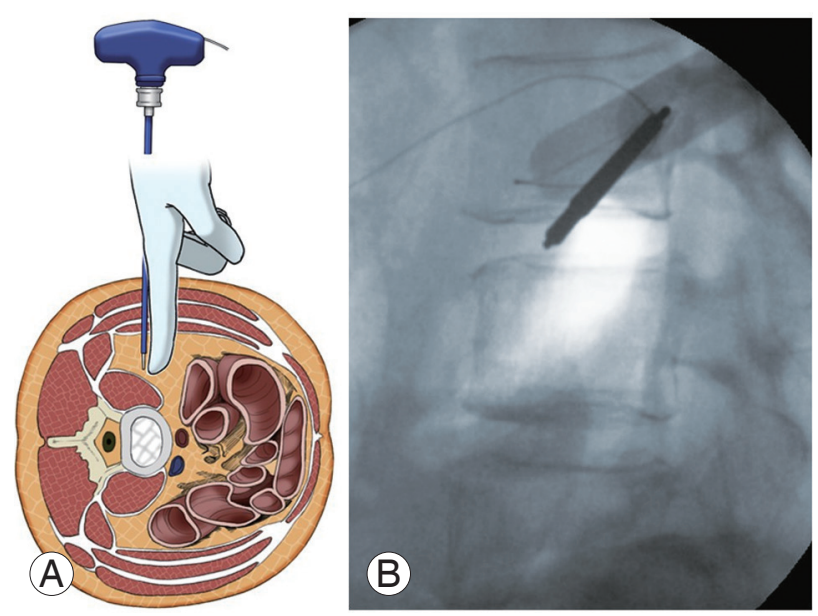

Fig. 5. With protection of peritoneal sac using a finger, a probe is inserted to the disc through a transpsoas approach (A). Next, lateral fluoroscopy is used to confirm the correct trajectory (B).

plate violation. Next, pituitary rongeur is used to remove the free disc fragments. After endplate preparation using a ring curette, an interbody graft packed with the surgeon's choice of biologics is inserted into the disc space. The operating table is leveled to relieve the tension of abdominal wall for skin closure.

\section{Pearls and pitfalls}

MIS-LLIF has shown promising outcomes in minimizing the approach-related morbidity. It can minimize soft tissue injury, decrease blood loss, and shorten hospital stay while maintaining the equivalent or improved clinical and radiographic outcomes as compared to the open procedures [67-69]. Sagittal and coronal deformity correction can be performed with large and lordotic cages [70-74]. Additionally, LLIF has been shown to increase the height of foramen and the surface of central canal, thus achieving indirect decompression $[73,75,76]$. However, the disadvantages of LLIF include injury to the psoas muscle, lumbar plexus, or bowel [74,77]. Approach-sided lower extremity weakness and paresthesia can also occur [74,77]. Furthermore, researchers have also reported incisional hernia and vascular injury [78].

The LLIF procedure is suitable for degenerative diseases that require the restoration of the disc height [56]. Moreover, the correction of spondylolisthesis or scoliosis is one of the most popular indications [70,73,79]. LLIF has particular advantages in the revision cases such as adjacent segment disease or post-laminectomy disease, wherein an open decompression has a high durotomy rate $[74,80]$. Contraindications are mostly secondary conditions that impede safe lateral approaches, such as prior retroperitoneal surgery, infection, or radiation therapy history. The L5-S1 disc is a contraindication to LLIF because the iliac wing precludes its lateral access [10]. In high-grade spondylolisthesis, the more anterior exiting nerve root is traversed horizontally, the more it is difficult to place the interbody graft. LLIF is also contraindicated if direct decompression is required, such as congenital stenosis, lateral recess stenosis, or sequestrated disc [10].

\section{Oblique Lumbar Interbody Fusion}

\section{Surgical techniques}

OLIF is an alternative to LLIF to overcome its complications. It accesses the intervertebral disc space between the psoas muscle and the aorta/inferior vena cava (IVC) to reduce the risk of injury to muscle and lumbar plexus. Therefore, surgeons should evaluate whether the width of the bare area is sufficient for OLIF on the preoperative imaging studies. Mayer first described OLIF, also known as anterior-to-psoas or prepsoas, in 1997 [81]. Patient positioning and setup for OLIF are nearly same to those for LLIF; however, there are some differences. Although LLIF allows either left- or right-side approaches, OLIF 


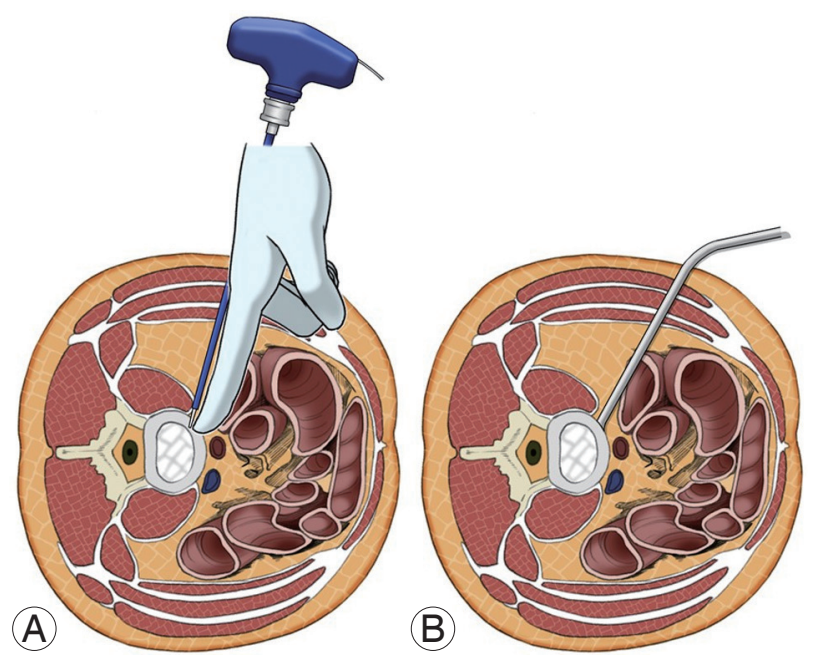

Fig. 6. An oblique corridor to access the disc space: a probe (A) and retractors (B) are placed between abdominal aorta and the psoas muscle.

should use a left-side approach because a working passage between IVC and psoas muscle is much narrow and risky than a right-side approach [82]. Transverse skin incisions are made centered on the anterior margin of the disc. The blunt dissection of abdominal wall and retroperitoneal space is same as those for LLIF. The oblique working corridor is established by the anterior retraction of the peritoneum and slight posterior retraction of the psoas muscle (Fig. 6). Because lumbar plexus is located within the psoas muscle, neuromonitoring is not needed [66]. The next sequential steps are similar to those of LLIF. However, any instruments used during OLIF should be introduced into the disc with the orthogonal maneuver to avoid the injury of contralateral nerve root.

\section{Pearls and pitfalls}

One of the important advantages of OLIF is the lack of any specific need for intraoperative neuromonitoring [66]. Moreover, the psoas muscle injury can be avoided. The surgical oblique view during OLIF is much favorable for the surgeons as compared to LLIF, which only allows a vertical view. Similar to LLIF, OLIF can provide indirect neural decompression and deformity correction $[29,56,83,84]$. Meta-analyses have showed that the risks of ipsilateral hip flexor weakness, transient thigh pain, and lumbar plexus injury are lower in OLIF than in LLIF $[77,85,86]$. However, the risk of vascular injury can increase because of the proximity of OLIF to the vessels as compared to LLIF $[77,86]$. Another complication of OLIF is sympathetic injury because sympathetic chains exit on the working window $[77,86]$. One of the key differences is the anatomical accessibility to L5-S1. Although LLIF is contraindicated, OLIF can target L5-S1.

Indications and contraindications are similar to those for LLIF. OLIF is suitable for degenerative lumbar diseases including sagittal and coronal deformity, similar to LLIF. Revision surgery is also one of the most popular surgical indications. OLIF is not suitable if direct decompression is required.

\section{Endoscopic Lumbar Interbody Fusion}

The trend toward MIS is widespread among all the surgical specialties, including spine surgery [87]. MIS spine surgery has the advantages of an early recovery and the preservation of normal structures. Therefore, MIS appears to be available for elderly and comorbid patients who are at an increased risk of morbidity with traditional open spine surgery. Therefore, spine surgeons have recently developed more advanced and less invasive techniques. With both the advancement of technology and anatomical understanding (i.e., Kambin's triangle), the endoscopic spine surgery has emerged. Recently, researchers have attempted endoscopic LIF procedures [88]. The most common and well-described endoscopic LIF is the TLIF [89], which is discussed in this section.

\section{Surgical techniques}

Endoscopic TLIF is performed using the biportal endoscopy systems, which include a console, camera, endoscopy irrigation equipment, and tool kits [88,90]. Additionally, a radiofrequency $(\mathrm{RF})$ console and RF probes are needed for tissue cauterization and bleeding control. The patient positioning is same as those for PLIF or TLIF. Two paramedian skin incision are made over the ipsilateral pedicles [91]. Two channels, one working and one endoscopic channel, are created through these two skin incisions. The ipsilateral laminectomy and facetectomy are performed using drill, Kerrison punches, or osteotomes. After the ligamentum flavum is removed, the ipsilateral nerve roots can be visualized. The next steps are similar to those of TLIF. Discectomy and endplate preparation are performed with the aid of the endoscopic channel. The autograft and interbody cage are placed through the working portal. Finally, the percutaneous pedicle screws are inserted bilaterally. 


\section{Pearls and pitfalls}

Because endoscopic LIF has been recently introduced, there is still not enough evidence about the pros and cons of its surgical outcomes and complications. Previous studies have reported promising postoperative outcomes [9193]. Some reports have shown that endoscopic LIF yielded lesser postoperative pain and shorter hospital stay than MIS-TLIF [94]. Moreover, despite the benefits of endoscopic LIF, there are inevitable obstacles to overcome and surgeons should reach the learning curve [92,95-97].

Considering a benefit of endoscopic LIF based on the previous reports, proper indications include patients, particularly the elderly, who are unable to tolerate open fusion surgery because of the risk of complications [92]. Clinical indications/contraindications for endoscopic LIF are evolving, but are now thought to be the same as those for MIS-fusion surgery [92].

\section{Conclusions}

LIF has been proven to be an effective treatment option for various spinal pathologies. Several approaches for LIF such as PLIF, TLIF, ALIF, LLIF, OLIF, and endoscopic LIF are available in the medical field. Each approach has its own pearls and pitfalls, and the surgeons should acknowledge these aspects before their practices. The optimal approach should be chosen based on the individual pathology, the anatomy of the patient, and the familiarity of the surgeon with available options. This review provides a broad review about the surgical procedures, advantages, disadvantages, and indications/contraindications of each LIF techniques. High-level evidence is also needed to help in decision-making when fusion is indicated.

\section{Conflict of Interest}

No potential conflict of interest relevant to this article was reported.

\section{Acknowledgments}

This work was supported by Small Grant for Exploratory Research (SGER) through the Ministry of Education of the Republic of Korea (grand no., 2018R1D1A1A02086045). The authors thank Suk-Woo Lee for illustrations in the manuscript.

\section{References}

1. De Kunder SL, Rijkers K, Caelers IJ, de Bie RA, Koehler PJ, van Santbrink H. Lumbar interbody fusion: a historical overview and a future perspective. Spine (Phila Pa 1976) 2018;43:1161-8.

2. Briggs $H$, Milligan PR. Chip fusion of the low back following exploration of the spinal canal. J Bone Joint Surg 1944;26:125-30.

3. Verma R, Virk S, Qureshi S. Interbody fusions in the lumbar spine: a review. HSS J 2020;16:162-7.

4. Cloward RB. The treatment of ruptured lumbar intervertebral discs by vertebral body fusion. I. Indications, operative technique, after care. J Neurosurg 1953;10:154-68.

5. Steffee AD, Sitkowski DJ. Posterior lumbar interbody fusion and plates. Clin Orthop Relat Res 1988;227:99102.

6. Harms J, Rolinger H. A one-stager procedure in operative treatment of spondylolistheses: dorsal traction-reposition and anterior fusion. Z Orthop Ihre Grenzgeb 1982;120:343-7.

7. Oppenheimer JH, DeCastro I, McDonnell DE. Minimally invasive spine technology and minimally invasive spine surgery: a historical review. Neurosurg Focus 2009;27:E9.

8. Eck JC, Hodges S, Humphreys SC. Minimally invasive lumbar spinal fusion. J Am Acad Orthop Surg 2007;15:321-9.

9. DiPaola CP, Molinari RW. Posterior lumbar interbody fusion. J Am Acad Orthop Surg 2008;16:130-9.

10. Garfin SR, Eismont FJ, Bell GR, Fischgrund JS, Bono CM. Rothman-Simeone and Herkowitz's the spine. 7th ed. Philadelphia (PA): Elsevier; 2018.

11. Lestini WF, Fulghum JS, Whitehurst LA. Lumbar spinal fusion: advantages of posterior lumbar interbody fusion. Surg Technol Int 1994;3:577-90.

12. Kim KT, Lee SH, Lee YH, Bae SC, Suk KS. Clinical outcomes of 3 fusion methods through the posterior approach in the lumbar spine. Spine (Phila Pa 1976) 2006;31:1351-7.

13. Fan SW, Hu ZJ, Fang XQ, Zhao FD, Huang Y, Yu $\mathrm{HJ}$. Comparison of paraspinal muscle injury in onelevel lumbar posterior inter-body fusion: modified minimally invasive and traditional open approaches. Orthop Surg 2010;2:194-200.

14. Okuda S, Miyauchi A, Oda T, Haku T, Yamamoto T, 
Iwasaki M. Surgical complications of posterior lumbar interbody fusion with total facetectomy in 251 patients. J Neurosurg Spine 2006;4:304-9.

15. Krishna M, Pollock RD, Bhatia C. Incidence, etiology, classification, and management of neuralgia after posterior lumbar interbody fusion surgery in 226 patients. Spine J 2008;8:374-9.

16. Humphreys SC, Hodges SD, Patwardhan AG, Eck JC, Murphy RB, Covington LA. Comparison of posterior and transforaminal approaches to lumbar interbody fusion. Spine (Phila Pa 1976) 2001;26:567-71.

17. Zhang Q, Yuan Z, Zhou M, Liu H, Xu Y, Ren Y. A comparison of posterior lumbar interbody fusion and transforaminal lumbar interbody fusion: a literature review and meta-analysis. BMC Musculoskelet Disord 2014;15:367.

18. Molinari RW, Gerlinger T. Functional outcomes of instrumented posterior lumbar interbody fusion in active-duty US servicemen: a comparison with nonoperative management. Spine J 2001;1:215-24.

19. Mobbs RJ, Phan K, Malham G, Seex K, Rao PJ. Lumbar interbody fusion: techniques, indications and comparison of interbody fusion options including PLIF, TLIF, MI-TLIF, OLIF/ATP, LLIF and ALIF. J Spine Surg 2015;1:2-18.

20. Foley KT, Lefkowitz MA. Advances in minimally invasive spine surgery. Clin Neurosurg 2002;49:499517.

21. Foley KT, Holly LT, Schwender JD. Minimally invasive lumbar fusion. Spine (Phila Pa 1976) 2003;28(15 Suppl):S26-35.

22. Lee MJ, Mok J, Patel P. Transforaminal lumbar interbody fusion: traditional open versus minimally invasive techniques. J Am Acad Orthop Surg 2018;26:124-31.

23. De Kunder SL, van Kuijk SM, Rijkers K, et al. Transforaminal lumbar interbody fusion (TLIF) versus posterior lumbar interbody fusion (PLIF) in lumbar spondylolisthesis: a systematic review and metaanalysis. Spine J 2017;17:1712-21.

24. Xu H, Tang H, Guan X, et al. Biomechanical comparison of posterior lumbar interbody fusion and transforaminal lumbar interbody fusion by finite element analysis. Neurosurgery 2013;72(1 Suppl Operative):21-6.

25. Sim HB, Murovic JA, Cho BY, Lim TJ, Park J. Biomechanical comparison of single-level posterior versus transforaminal lumbar interbody fusions with bilateral pedicle screw fixation: segmental stability and the effects on adjacent motion segments. J Neurosurg Spine 2010;12:700-8.

26. AlShazli AB, Amer AY, Sultan AM, et al. Minimally invasive transforaminal lumbar interbody fusion for the surgical management of post-discectomy syndrome. Asian Spine J 2020;14:148-56.

27. Hammad A, Wirries A, Ardeshiri A, Nikiforov O, Geiger F. Open versus minimally invasive TLIF: literature review and meta-analysis. J Orthop Surg Res 2019;14:229.

28. Khechen B, Haws BE, Patel DV, et al. Comparison of postoperative outcomes between primary MIS TLIF and MIS TLIF with revision decompression. Spine (Phila Pa 1976) 2019;44:150-6.

29. Champagne PO, Walsh C, Diabira J, et al. Sagittal balance correction following lumbar interbody fusion: a comparison of the three approaches. Asian Spine J 2019;13:450-8.

30. Kim CH, Easley K, Lee JS, et al. Comparison of minimally invasive versus open transforaminal interbody lumbar fusion. Global Spine J 2020;10(2 Suppl):143S150 S.

31. Terman SW, Yee TJ, Lau D, Khan AA, La Marca F, Park P. Minimally invasive versus open transforaminal lumbar interbody fusion: comparison of clinical outcomes among obese patients. J Neurosurg Spine 2014;20:644-52.

32. Wong AP, Smith ZA, Stadler JA 3rd, et al. Minimally invasive transforaminal lumbar interbody fusion (MI-TLIF): surgical technique, long-term 4-year prospective outcomes, and complications compared with an open TLIF cohort. Neurosurg Clin N Am 2014;25:279-304.

33. Tian NF, Wu YS, Zhang XL, Xu HZ, Chi YL, Mao FM. Minimally invasive versus open transforaminal lumbar interbody fusion: a meta-analysis based on the current evidence. Eur Spine J 2013;22:1741-9.

34. Vazan M, Gempt J, Meyer B, Buchmann N, Ryang YM. Minimally invasive transforaminal lumbar interbody fusion versus open transforaminal lumbar interbody fusion: a technical description and review of the literature. Acta Neurochir (Wien) 2017;159:113746.

35. Javernick MA, Kuklo TR, Polly DW Jr. Transforaminal lumbar interbody fusion: unilateral versus 
bilateral disk removal: an in vivo study. Am J Orthop (Belle Mead NJ) 2003;32:344-8.

36. McAfee PC, Lee GA, Fedder IL, Cunningham BW. Anterior BAK instrumentation and fusion: complete versus partial discectomy. Clin Orthop Relat Res 2002;(394):55-63.

37. Sclafani JA, Kim CW. Complications associated with the initial learning curve of minimally invasive spine surgery: a systematic review. Clin Orthop Relat Res 2014;472:1711-7.

38. Nandyala SV, Fineberg SJ, Pelton M, Singh K. Minimally invasive transforaminal lumbar interbody fusion: one surgeon's learning curve. Spine J 2014;14:1460-5.

39. Lee JC, Jang HD, Shin BJ. Learning curve and clinical outcomes of minimally invasive transforaminal lumbar interbody fusion: our experience in 86 consecutive cases. Spine (Phila Pa 1976) 2012;37:1548-57.

40. Choi J, Rhee I, Ruparel S. Assessment of great vessels for anterior access of L5/S1 using patient positioning. Asian Spine J 2020;14:438-44.

41. Baker JF, Chan JC, Moon BG, Robertson PA. Relationship of aortic bifurcation with sacropelvic anatomy: application to anterior lumbar interbody fusion. Clin Anat 2020 Apr 5 [Epub]. https://doi. org/10.1002/ca.23598.

42. Liu L, Liang Y, Zhou Q, et al. Study on the anatomy of the lumbosacral anterior great vessels pertinent to L5/S1 anterior interbody surgery with computer tomography angiography. Acta Orthop Belg 2014;80:537-43.

43. Bridwell KH, Gupta M. Bridwell and DeWald's textbook of spinal surgery. 4th ed. Philadelphia (PA): Wolters Kluwer Health; 2019.

44. Sasso RC, Kenneth Burkus J, LeHuec JC. Retrograde ejaculation after anterior lumbar interbody fusion: transperitoneal versus retroperitoneal exposure. Spine (Phila Pa 1976) 2003;28:1023-6.

45. Gumbs AA, Bloom ND, Bitan FD, Hanan SH. Open anterior approaches for lumbar spine procedures. Am J Surg 2007;194:98-102.

46. Lolis E, Panagouli E, Venieratos D. Study of the ascending lumbar and iliolumbar veins: surgical anatomy, clinical implications and review of the literature. Ann Anat 2011;193:516-29.

47. Bassani R, Morselli C, Querenghi AM, Nuara A, Sconfienza LM, Peretti GM. Functional and radio- logical outcome of anterior retroperitoneal versus posterior transforaminal interbody fusion in the management of single-level lumbar degenerative disease. Neurosurg Focus 2020;49:E2.

48. Allain J, Dufour T. Anterior lumbar fusion techniques: ALIF, OLIF, DLIF, LLIF, IXLIF. Orthop Traumatol Surg Res 2020;106:S149-57.

49. Upadhyayula PS, Curtis EI, Yue JK, Sidhu N, Ciacci JD. Anterior versus transforaminal lumbar interbody fusion: perioperative risk factors and 30-day outcomes. Int J Spine Surg 2018;12:533-42.

50. Sihvonen T, Herno A, Paljarvi L, Airaksinen O, Partanen J, Tapaninaho A. Local denervation atrophy of paraspinal muscles in postoperative failed back syndrome. Spine (Phila Pa 1976) 1993;18:575-81.

51. Qureshi R, Puvanesarajah V, Jain A, Shimer AL, Shen $\mathrm{FH}$, Hassanzadeh H. A comparison of anterior and posterior lumbar interbody fusions: complications, readmissions, discharge dispositions, and costs. Spine (Phila Pa 1976) 2017;42:1865-70.

52. Bateman DK, Millhouse PW, Shahi N, et al. Anterior lumbar spine surgery: a systematic review and meta-analysis of associated complications. Spine J 2015;15:1118-32.

53. Xu DS, Walker CT, Godzik J, Turner JD, Smith W, Uribe JS. Minimally invasive anterior, lateral, and oblique lumbar interbody fusion: a literature review. Ann Transl Med 2018;6:104.

54. Teng I, Han J, Phan K, Mobbs R. A meta-analysis comparing ALIF, PLIF, TLIF and LLIF. J Clin Neurosci 2017;44:11-7.

55. Lee N, Kim KN, Yi S, et al. Comparison of outcomes of anterior, posterior, and transforaminal lumbar interbody fusion surgery at a single lumbar level with degenerative spinal disease. World Neurosurg 2017;101:216-26.

56. Cho JY, Goh TS, Son SM, Kim DS, Lee JS. Comparison of anterior approach and posterior approach to instrumented interbody fusion for spondylolisthesis: a meta-analysis. World Neurosurg 2019;129:e286-93.

57. Anand N, Alayan A, Agrawal A, Kahwaty S, Nomoto E, Khandehroo B. Analysis of spino-pelvic parameters and segmental lordosis with L5-S1 oblique lateral interbody fusion at the bottom of a long construct in circumferential minimally invasive surgical correction of adult spinal deformity. World Neurosurg 2019;130:e1077-83. 
58. Watkins RG 4th, Hanna R, Chang D, Watkins RG 3rd. Sagittal alignment after lumbar interbody fusion: comparing anterior, lateral, and transforaminal approaches. J Spinal Disord Tech 2014;27:253-6.

59. Lee CS, Park SJ, Chung SS, Lee JY, Yum TH, Shin SK. Mini-open anterior lumbar interbody fusion combined with lateral lumbar interbody fusion in corrective surgery for adult spinal deformity. Asian Spine J 2016;10:1023-32.

60. Manunga J, Alcala C, Smith J, et al. Technical approach, outcomes, and exposure-related complications in patients undergoing anterior lumbar interbody fusion. J Vasc Surg 2020 Jul 21 [Epub]. https:// doi.org/10.1016/j.jvs.2020.06.129.

61. Lindley EM, McBeth ZL, Henry SE, et al. Retrograde ejaculation after anterior lumbar spine surgery. Spine (Phila Pa 1976) 2012;37:1785-9.

62. Comer GC, Smith MW, Hurwitz EL, Mitsunaga KA, Kessler R, Carragee EJ. Retrograde ejaculation after anterior lumbar interbody fusion with and without bone morphogenetic protein-2 augmentation: a 10year cohort controlled study. Spine J 2012;12:881-90.

63. Mobbs RJ, Phan K, Daly D, Rao PJ, Lennox A. Approach-related complications of anterior lumbar interbody fusion: results of a combined spine and vascular surgical team. Global Spine J 2016;6:147-54.

64. Ozgur BM, Aryan HE, Pimenta L, Taylor WR. Extreme lateral interbody fusion (XLIF): a novel surgical technique for anterior lumbar interbody fusion. Spine J 2006;6:435-43.

65. Riley MR, Doan AT, Vogel RW, Aguirre AO, Pieri KS, Scheid EH. Use of motor evoked potentials during lateral lumbar interbody fusion reduces postoperative deficits. Spine J 2018;18:1763-78.

66. Guerin P, Obeid I, Bourghli A, et al. The lumbosacral plexus: anatomic considerations for minimally invasive retroperitoneal transpsoas approach. Surg Radiol Anat 2012;34:151-7.

67. Nakashima H, Kanemura T, Satake K, et al. Comparative radiographic outcomes of lateral and posterior lumbar interbody fusion in the treatment of degenerative lumbar kyphosis. Asian Spine J 2019;13:395402.

68. Nakashima H, Kanemura T, Satake K, et al. Changes in sagittal alignment following short-level lumbar interbody fusion: comparison between posterior and lateral lumbar interbody fusions. Asian Spine J
2019;13:904-12.

69. Derman PB, Albert TJ. Interbody fusion techniques in the surgical management of degenerative lumbar spondylolisthesis. Curr Rev Musculoskelet Med 2017;10:530-8.

70. Iwamae M, Matsumura A, Namikawa T, et al. Surgical outcomes of multilevel posterior lumbar interbody fusion versus lateral lumbar interbody fusion for the correction of adult spinal deformity: a comparative clinical study. Asian Spine J 2020;14:421-9.

71. Nakashima H, Kanemura T, Satake K, et al. Factors affecting postoperative sagittal alignment after lateral lumbar interbody fusion in adult spinal deformity: posterior osteotomy, anterior longitudinal ligament rupture, and endplate injury. Asian Spine J 2019;13:738-45.

72. Laws CJ, Coughlin DG, Lotz JC, Serhan HA, Hu SS. Direct lateral approach to lumbar fusion is a biomechanically equivalent alternative to the anterior approach: an in vitro study. Spine (Phila $\mathrm{Pa} 1976$ ) 2012;37:819-25.

73. Taba HA, Williams SK. Lateral lumbar interbody fusion. Neurosurg Clin N Am 2020;31:33-42.

74. Park HY, Kim YH, Ha KY, et al. Minimally invasive lateral lumbar interbody fusion for clinical adjacent segment pathology: a comparative study with conventional posterior lumbar interbody fusion. Clin Spine Surg 2019;32:E426-33.

75. Rabau O, Navarro-Ramirez R, Aziz M, et al. Lateral lumbar interbody fusion (LLIF): an update. Global Spine J 2020;10(2 Suppl):17S-21S.

76. Limthongkul W, Tanasansomboon T, Yingsakmongkol W, Tanaviriyachai T, Radcliff K, Singhatanadgige W. Indirect decompression effect to central canal and ligamentum flavum after extreme lateral lumbar interbody fusion and oblique lumbar interbody fusion. Spine (Phila Pa 1976) 2020;45:E1077-84.

77. Walker CT, Farber SH, Cole TS, et al. Complications for minimally invasive lateral interbody arthrodesis: a systematic review and meta-analysis comparing prepsoas and transpsoas approaches. J Neurosurg Spine 2019:1-15.

78. Vivas AC, Januszewski J, Hajirawala L, Paluzzi JM, Gandhi SV, Uribe JS. Incisional hernia after minimally invasive lateral retroperitoneal surgery: case series and review of the literature. Oper Neurosurg (Hagerstown) 2019;16:368-73. 
79. McGowan JE, Kanter AS. Lateral approaches for the surgical treatment of lumbar spondylolisthesis. Neurosurg Clin N Am 2019;30:313-22.

80. Kudo Y, Okano I, Toyone T, et al. Lateral lumbar interbody fusion in revision surgery for restenosis after posterior decompression. Neurosurg Focus 2020;49:E11.

81. Mayer HM. A new microsurgical technique for minimally invasive anterior lumbar interbody fusion. Spine (Phila Pa 1976) 1997;22:691-9.

82. Hu WK, He SS, Zhang SC, et al. An MRI study of psoas major and abdominal large vessels with respect to the X/DLIF approach. Eur Spine J 2011;20:557-62.

83. Beng TB, Kotani Y, Sia U, Gonchar I. Effect of indirect neural decompression with oblique lateral interbody fusion was influenced by preoperative lumbar lordosis in adult spinal deformity surgery. Asian Spine J 2019;13:809-14.

84. Kim WJ, Lee JW, Park KY, Chang SH, Song DG, Choy WS. Treatment of adult spinal deformity with sagittal imbalance using oblique lumbar interbody fusion: can we predict how much lordosis correction is possible? Asian Spine J 2019;13:1017-27.

85. Kim WJ, Lee JW, Kim SM, et al. Precautions for combined anterior and posterior long-level fusion for adult spinal deformity: perioperative surgical complications related to the anterior procedure (oblique lumbar interbody fusion). Asian Spine J 2019;13:82331.

86. Spiessberger A, Arvind V, Dietz N, et al. A comparison of complications and clinical and radiologic outcome between the mini-open prepsoas and miniopen transpsoas approaches for lumbar interbody fusion: a meta-analysis. Clin Spine Surg 2020;33:2719.

87. Patel PD, Canseco JA, Houlihan N, Gabay A, Grasso G, Vaccaro AR. Overview of minimally invasive spine surgery. World Neurosurg 2020;142:43-56.

88. Kim JE, Choi DJ, Park EJ, et al. Biportal endoscopic spinal surgery for lumbar spinal stenosis. Asian Spine J 2019;13:334-42.
89. Kapetanakis S, Gkantsinikoudis N, Thomaidis T, Charitoudis G, Theodosiadis P. The role of percutaneous transforaminal endoscopic surgery in lateral recess stenosis in elderly patients. Asian Spine J 2019;13:638-47.

90. Heo DH, Hong YH, Lee DC, Chung HJ, Park CK. Technique of biportal endoscopic transforaminal lumbar interbody fusion. Neurospine 2020;17(Suppl 1):S129-37.

91. Heo DH, Son SK, Eum JH, Park CK. Fully endoscopic lumbar interbody fusion using a percutaneous unilateral biportal endoscopic technique: technical note and preliminary clinical results. Neurosurg Focus 2017;43:E8.

92. Brusko GD, Wang MY. Endoscopic lumbar interbody fusion. Neurosurg Clin N Am 2020;31:17-24.

93. Lee SH, Erken HY, Bae J. Percutaneous transforaminal endoscopic lumbar interbody fusion: clinical and radiological results of mean 46-month follow-up. Biomed Res Int 2017;2017:3731983.

94. Kim JE, Yoo HS, Choi DJ, Park EJ, Jee SM. Comparison of minimal invasive versus biportal endoscopic transforaminal lumbar interbody fusion for single-level lumbar disease. Clin Spine Surg 2020 Jun 1 [Epub]. https://doi.org/10.1097/ BSD.0000000000001024.

95. Choi DJ, Choi CM, Jung JT, Lee SJ, Kim YS. Learning curve associated with complications in biportal endoscopic spinal surgery: challenges and strategies. Asian Spine J 2016;10:624-9.

96. Yang J, Guo C, Kong Q, et al. Learning curve and clinical outcomes of percutaneous endoscopic transforaminal decompression for lumbar spinal stenosis. Int Orthop 2020;44:309-17.

97. Kolcun JP, Brusko GD, Basil GW, Epstein R, Wang MY. Endoscopic transforaminal lumbar interbody fusion without general anesthesia: operative and clinical outcomes in 100 consecutive patients with a minimum 1-year follow-up. Neurosurg Focus 2019;46:E14. 